\title{
Application Modes of Egress Simulation
}

\author{
S.M.V.Gwynne ${ }^{1}$, and E.D.Kuligowski ${ }^{2}$ \\ ${ }^{1}$ Hughes Associates, Inc., USA \\ Contact email: sgwynne@haifire.com \\ 2 National Institute of Standards and Technology, USA \\ Contact email: erica.kuligowski@nist.gov
}

Summary. Egress models are being used more frequently to simulate people movement; i.e. how people enter, use and leave a building. However, little has been written on the different aspects of people movement that can be examined and how these models may achieve this. This paper outlines six modes in which an egress model can be applied: Nä̈ve; Operational; Predictive; Engineered; Real-Time; and Interactive. The paper outlines what is needed to enable these application modes, in terms of data, expertise and model functionality, and the benefits of doing so. This is intended to highlight the challenges faced by egress models and the complexities of the subject matter being examined: people movement under emergency and nonemergency scenarios. Currently, no model includes all of the six modes identified. The authors hope that this discussion will identify the importance of these modes, the need for them to be addressed within the same model and the clear benefits of doing so.

\section{Introduction}

Computational egress models have grown more sophisticated and are now frequently used as part of a performance-based assessment. This development has followed an increase in our understanding of human behavior in fire and a modest increase in the supporting empirical data available. The model developments have responded to this increased understanding, albeit in an imperfect, delayed and inconsistent manner. This has allowed computational egress models to be applied to new and more complex application areas. This has both increased the overall application of egress models and prompted a more rigorous examination of the functionality of the models in question. In addition, this increased breadth and depth of application requires a greater degree of expertise from the user and more comprehensive and reliable supporting data.

The expansion of the modeling capability poses questions regarding new application opportunities. This paper describes the different application modes of egress models; i.e. what the modes require of the computational models, the user, the data and the applications that they enable. This will provide a clearer understanding of the capabilities and limitations of the models, the expertise required of the user and the increasing demand for new, more comprehensive and more detailed data to the development and application of these models. 


\section{People Movement System (ICE)}

A building can be seen as a people movement system that operates at different states [1]. This system is formed from three phases of people movement (ICE): ingress; circulation; and egress. These phases of movement can exist under emergency and non-emergency conditions. Each of these phases will have informal or formal procedures to manage their effectiveness; e.g. security, emergency evacuation, etc. All forms of movement in relation to the structure (i.e. procedural responses), will fall into one of the six combinations, as shown in Table 1 .

Models can be applied to assess the effectiveness of these procedures. Each combination of phase and scenario requires a different set of assumptions and modeling capabilities.

Table 1: Examples of the procedures employed given the phase of movement and the scenario. [1]

\begin{tabular}{|c|c|c|c|}
\hline \multirow{2}{*}{ ICE } & \multicolumn{2}{c|}{ Scenario } \\
\cline { 2 - 4 } & $\begin{array}{c}\text { Ingress } \\
\text { (I) }\end{array}$ & $\begin{array}{c}\text { Crowd management, Fire } \\
\text { Department Arrival }\end{array}$ & $\begin{array}{c}\text { Security, Ticketed } \\
\text { Access }\end{array}$ \\
\cline { 2 - 4 } $\begin{array}{c}\text { Phase of } \\
\text { Movement }\end{array}$ & $\begin{array}{c}\text { Circulation } \\
\text { (C) }\end{array}$ & $\begin{array}{c}\text { Ensuring initiation of } \\
\text { response, disengagement of } \\
\text { population from activities }\end{array}$ & $\begin{array}{c}\text { Providing information } \\
\text { on facilities and } \\
\text { services }\end{array}$ \\
\cline { 2 - 4 } & $\begin{array}{c}\text { Egress } \\
\text { (E) }\end{array}$ & $\begin{array}{c}\text { Managing emergency } \\
\text { evacuation }\end{array}$ & $\begin{array}{c}\text { Leaving the building, } \\
\text { crowd management }\end{array}$ \\
\hline
\end{tabular}

Also, an incident does not occur independently of previous events: the prior use of a structure will influence the current event [1,2]. Therefore, in addition to the procedural efforts to manage people movement, these other historical factors will also have an impact on occupant behavior. Procedural and historical influences will therefore coexist. The history of the structure's use will be stored in occupant recollections (see Figure 1). These occupants will have a base knowledge and level of experience reflecting their previous use of the building and related training. They will also be provided with new information during any event. Procedural and historical factors will influence their activities during any of the phases of movement highlighted.

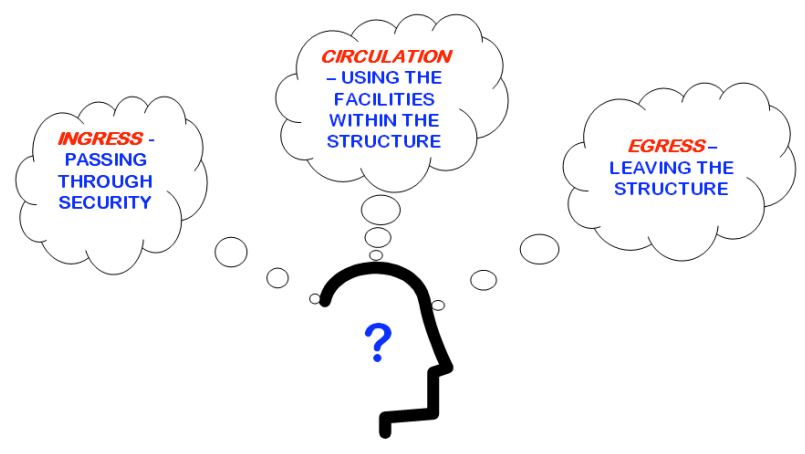

Figure 1: The individual brings their history with them to the evacuation. 
These procedural and historical factors are highly coupled: coexisting procedures can influence each other; an individual's experience will influence their behavior and their response to a procedure [1,2]. The application of one procedure (e.g. managing security) may influence the effectiveness of another (e.g. evacuation). This may occur directly (e.g. congestion produced by security delays evacuation movement) or indirectly (e.g. where an individual's prior restricted access due to security inhibits their use of certain exits). This can have an enormous influence over the performance of the people movement system. This performance, and our assessment of it, is critical, especially when making design decisions for emergency scenarios.

Computational models are increasingly employed to examine the people movement system. Given the highly coupled nature of the procedural and historical factors, it is contended that for an egress model to accurately represent any phase of movement, that it should ideally be able to represent all of them. This would then allow the model to capture the indirect and direct influences of these factors upon the results produced. If this is not possible then the results produced by a model may be quantitatively inaccurate (e.g. the evacuation times produced are too optimistic) and qualitatively inaccurate (e.g. congestion is not produced, exit use is unrealistic, etc.). This may lead to flawed and potentially unsafe design.

Sophisticated models are therefore required. These models will need to cope with the range of influences, situations and applications highlighted above. In the next sections, six different application modes are described. Each of these provides a means to estimate a different set of results or assess a different phase of people movement. It is contended that each of these modes provides an invaluable insight into the people movement system; it is also contended that to gain reliable insight into any single phase of people movement, that all of these modes should be available in the same computational model.

\section{Six Degrees of Simulation}

In the following sections, six simulation modes are characterized: Nä̈ve; Operational; Predictive; Engineered; Real-Time; and Interactive. Each is described in detail outlining the nature of each mode, the different demands placed on the user, the assessments made possible by each mode, and the data required to support the use of the mode.

\subsection{Naïve Mode}

Nä̈ve mode establishes movement patterns within a structure prior to the population's experience and knowledge levels developing; i.e. before the building design is put into practice. The model achieves this by simulating the movement of naïve occupants who gain experience and knowledge of the building by exposure to information as they move around the space. This movement could be biased or managed through the insertion of facilities, egress routes, signage, etc. In order for this mode to be possible within a simulation model, occupants start with no information or knowledge regarding the layout of the building. They initially move towards route elements that can be detected from their current position, or learn about the 
existence of such routes. General rules are used to govern the naïve movement of the individuals, although this movement evolves as more information becomes available to them. As this population becomes more informed, so their movement depends more on the routes available, their familiarity with these routes, and their typical use of these routes. This movement can be compiled and an understanding of the critical and most frequently used paths attained.

This mode can be used prior to the construction of a building or where the building has yet to be occupied. For instance, an engineer/architect/authority having jurisdiction (AHJ) may be interested in understanding how a shopping mall is used, given the positioning of certain facilities, signs and exits. The engineer can then modify the position of these facilities in order to improve the efficiency of the use of the space. This may have an impact on comfort levels, usage and on commercial viability.

The user would need to provide information on the location of the facilities, amenities, routes, information available, the structure, and the population characteristics. For each of the physical and informational components, the user would need to establish their relative impact on the knowledge levels of the population. The user would then need to understand the relationship between these physical and informational components, and the behavioral response of the population. The user would also need to identify critical events (e.g. congestion) and key components (e.g. exits, signs, etc.) that have an important impact on the use of the space. The results would require a high degree of interpretation; i.e. current engineering assessment criteria do not provide explicit guidance on this mode of application.

For a model to include this mode, it would need to represent the population at the individual level. The information levels available to each individual would need to be simulated; this information would need to be dynamic; and sensitive to the surrounding conditions. The structure would need to be represented in detail, including amenities and other elements that influence the individual's understanding of the structure. Behavioral algorithms would need to represent the impact of these amenities and elements upon the individual. The results produced would need to reflect the usage of certain elements, the information levels of the population, and the level of influence exerted by amenities and elements. This type of mode is currently available. The EVAS Pedestrian Modelling Software [3] produced by the University College London has a Nä̈ve mode. ${ }^{1}$

\subsection{Operational Mode}

Operational mode establishes the impact of 'normal' circulatory movement within a structure; i.e. how people routinely use the structure. This may be informed by the results produced in Nä̈ve mode; i.e. that the initial

1.

${ }^{1}$ Certain commercial entities, equipment, or materials may be identified in this document in order to describe an experimental procedure or concept adequately. Such identification is not intended to imply recommendation or endorsement by the National Institute of Standards and Technology, nor is it intended to imply that the entities, materials, or equipment are necessarily the best available for the purpose. 
conditions of Operational mode will be the results from Naïve mode. These results will influence the familiarity levels that people have with the space and with their preferred access routes. In the absence of these results, dedicated empirical data can be collected or engineering judgment regarding the use of the existing structure.

The model is applied in Operational mode by distributing the population about the space according to its use and then using the space as expected. In this case, the population will have a pre-determined understanding of the space and a set of actions that they would normally complete as part of their daily routine within the building. For instance, people's movement about an office-tower can be assessed given their office location, the location of the dinner hall, meeting rooms, etc. The movement patterns around the structure can then be simulated and areas of high use identified. This mode might be used to improve the efficiency of the usage of the building and also to improve the non-emergency management of the structure. It can also be used to understand the population's familiarity of the structure and also where the population might be at any one time. Both of these factors will directly influence the results produced during an emergency scenario.

Operational mode can be used after the structure's construction has been completed and when the building is in operation. It would allow further insight into the current use of the structure and into the impact of procedural and structural changes upon this use.

In order for Operational mode to function, it requires the initial distribution of the population (either from empirical data or from Naïve mode), the routes used during non-emergency movement, and the locations of key components within the structure. These requirements are similar to those of the Naïve mode except that the population is credited with having a previously established familiarity with the space.

In order to apply Operational mode, the user requires an understanding of how people make use of the structure in question, an understanding of nonemergency behavior in general in order to identify anomalous behaviors and the ability to interpret the results produced. This mode will require a high degree of interaction between the user and the model in order to configure the model for simulation and then to interpret the results produced.

A simulation model would need to represent a population as individuals and associate pre-defined, non-emergency tasks to them; e.g. visit cafeteria, go to bathroom, attend meeting, etc. These tasks might implicitly or explicitly be associated with the typical operations within the structure; e.g. the tasks can be specifically defined, or alternatively the time delay associated with the task can be represented. The simulated population would need to be sensitive to a range of influences upon their behavior. The model would need to simulate actions as well as movement, cope with a range of different procedures, and possibly generate people during simulation; e.g. the arrival of people at a rail station. 
A number of models are currently available that include this type of functionality: one example being the EXODUS model [4], which is able to simulate non-emergency activities that can then develop into an emergency scenario. The population can be given pre-determined activities as part of a non-emergency event. These activities are followed until an emergency arises, when these activities can potentially be ignored.

\subsection{Predictive Mode}

Predictive mode allows the user to investigate the impact of local evacuee decision-making on egress performance; i.e. to examine the outcome of an evacuation from the bottom-up rather than imposing a behavioral response. Rather than these behaviors being entirely pre-determined by the user, they instead occur through an adaptive process: low-level behaviors (e.g. delay, move, redirect, etc.) produce emergent high-level conditions (evacuate, seek refuge, etc.). The mode represents an attempt to genuinely predict the behavioral response, given the conditions faced, rather than simply to investigate the consequences of an entirely imposed response to an event.

The 'intelligence' is built into the model rather than residing in the engineer. For instance, instead of the engineer prescribing the response of the evacuating population (e.g. they respond between times $\mathrm{X}$ and $\mathrm{Y}$ and use exit $Z$ ), the behaviors are instead dependent upon local conditions, the actions of the surrounding population, the information available to the individual and their abilities. This reliance on the model's capabilities produces significant (and often warranted) levels of scepticism. The value of this approach is therefore highly sensitive to the sophistication of the behavioral model employed. However, the benefits of this approach do not necessarily rely on the location of the expertise (although the inconsistent understanding of human behavior in the field of engineering is certainly an issue). It instead relies on the ability to generate evacuee responses, rather than having them prescribed.

This mode can inform further scenario design for assessment; for instance, in a performance-based analysis the Predictive mode can be used to identify additional scenarios of interest. In typical engineering applications, a set of scenarios (questions) is posed, the model is set up to address these scenarios and then results are produced. The engineer configures and constrains the model to answer the questions posed; however, this does not ensure that the correct questions are being posed. By definition, the engineer prescribes the behaviors that can be performed during the simulation in order to focus on the questions being investigated; e.g. a set of pre-evacuation times, response to the presence of smoke or congestion, etc. that relate to these questions.

The use of the Predictive mode, where the simulated population is allowed to adapt to the scenario, may generate new questions, allow new worst-case scenarios to be developed (although given the limited time available the term 'worse-case' is more appropriate), and highlight new previously unforeseen conditions. These are made possible by the adaptive response of the evacuees; e.g. the pre-evacuation times might be generated by the presence of a type of alarm, the presence of smoke, the actions of others, etc., rather than 
being imposed by the engineer. These emergency conditions may allow the identification of informed worse-case scenarios, rather than those produced beforehand by the engineer. It can also be used to determine the underlying causes of observations: it allows a genuinely investigative process to take place. If the user predetermines evacuee responses then only these responses can be tested.

The Predictive mode requires the initial conditions to be provided in great detail, as these conditions will influence the behavioral response of the evacuees. These include the initial distribution of the population, the nature of the population, the scenario conditions, etc. These could be provided by the collection of empirical data or from earlier model calculations; e.g. the data provided by Operational mode. Critically, it also requires a comprehensive behavioral model that is sufficiently developed to provide the range of behaviors needed. This model will need to be based on an adaptive approach rather than a purely deterministic approach; i.e. it will need to be sensitive to the emergent qualities necessary for the Predictive mode. This will require the individually simulated evacuees assessing the surrounding conditions and then selecting their response accordingly. This adaptive process will represent the connection between external events and the actions performed by the individual.

The primary expertise required to employ this mode is in the configuration of the scenario, determining that the simulated behavioral responses are reasonable, and in the analysis of the results produced. The key behavioral decisions (i.e. the appropriateness and selection of a behavioral response to certain conditions) are taken within the model itself. As mentioned previously, the expertise required is embedded in the model itself by the model developers. This does not imply that the engineer is exempt from expertise in egress behaviour, as this is critical when configuring the model; only that this expertise is not employed in selecting evacuee behaviors. However, a great deal of expertise is required in examining the results and extracting the underlying factors and behaviors that influenced them. This is more complex as it is not always apparent that a direct relationship exists between a particular behavior and an outcome. This requires an understanding of the simulated conditions, the methods employed and the subject matter being simulated.

Currently, there are no fully predictive egress models. There are some models that include adaptive behaviors (such as the MASSEgress model [5]), but these are primarily research-based. Where these models are commercially available (see $[4,6]$ ), the adaptive capabilities tend, by default, to be disabled given that most applications require that the evacuee behavioral response is constrained; i.e. that the behavioral responses are controlled by the engineer. The absence of a fully predictive model is due to a number of reasons:

- the difficulty in producing such models; e.g. they are computationally expensive, and technically complex.

- the difficult in supporting their development with appropriate data; e.g. most data represents high-level behavioral responses, rather than the constituent components required for this mode to function. 
- the scepticism that such predictive modes face, given their emergent nature; e.g. it is more likely that an AHJ would trust an engineer that can explain his/her assumption regarding a scenario than rely on the documented theoretical assumptions in support of complex behavioral algorithms.

- $\quad$ and the nature of the current performance-based regulations; i.e. that they are relatively immature.

\subsection{Engineered Mode}

Engineered mode allows the user to pre-determine emergency scenarios and then impose them and the subsequent behavioral response upon an evacuating population; i.e. to examine the outcome of an evacuation from the top-down. This is the mode typically used by engineers to assess design performance. It allows specific questions to be posed and variables to be controlled within the simulation process to allow specific answers to be produced. This control allows the engineer to prescribe the behavioral response, reducing the number of confounding variables; e.g. evacuees respond between $\mathrm{X}$ and $\mathrm{Y}$ seconds and use exit $\mathrm{Z}$. This is very useful given the current performance-based regulations [7].

Engineered mode requires data-sets that address specific egress components, rather than those that support a comprehensive, behavioral model. Indeed, one of the key benefits of this mode is that it allows, to some degree, the engineer to account for the current limitations in the data available. The data employed will be model-specific, but will generally relate to the key egress components: the pre-evacuation time; the relationship between population density and flow; the movement rates for different sections of the population and different structural components; simple evacuee behaviors, such as route usage; and possibly the impact of certain impairments. This will then allow the model to simulate the required evacuee responses given the predetermined questions posed by the engineer.

The engineer will need to provide a description of the initial conditions: population, structure, procedure and environment. These may be informed by Operational mode. The scenarios examined may be informed by the results produced in the Predictive mode, where new 'worse-case' scenarios had previously been identified. The engineer is responsible for posing the correct questions and then configuring the model such that it can answer these questions. Given that the pre-determined questions are being addressed, the results produced can be examined to assess the outcome. Although some interpretation is required, it is certainly less than that required in the Predictive mode, and clearer guidance exists on what results need to be examined. However, expertise is still required. It is often the case that the results produced by simple models are presented as definitive, whereas they are, at best, suggestive [8].

To enable Engineered mode, a model must represent the required scenario conditions and allow the questions to be posed; i.e. allow the user to have control over the behavioral response and the scenario conditions. For instance, the engineer may need to have people respond instantaneously or 
according to a representative distribution; have them use their nearest exits or use exits according to familiarity; and include an unimpaired population and then a population that includes a section that has an impairment. A number of models are available to do such analysis (one example is Simulex [9]). In terms of fire safety engineering, this represents the most common form of analysis and the most commercially viable.

\subsection{Interactive Mode}

Interactive mode allows the engineer to modify aspects of the incident and/or the evacuee response during the simulation. It achieves this by allowing the engineer to interact with the simulation as it is running: to modify conditions and then have the model react to these changes. The engineer may act as a simulated evacuee (i.e. allowing them to take decisions for an evacuee) or as an 'overseer' (i.e. changing the scenario conditions to which the simulated evacuees are exposed and forced to react). The simulated conditions and/or evacuee responses are then modified by the engineer during the simulation. This mode allows the engineer to gain first-hand insight into the evacuation conditions, into the effectiveness of different evacuee responses, investigate worse-case scenarios, and/or be of value as a training/demonstration tool.

The engineer will have to initially configure the scenario conditions, as he/she would have in Engineered mode. However, additional data may have to be provided given the evolution of the scenario in response to the engineer's intervention. The engineer will also need to know how to use such a mode responsibly; e.g. to respond reasonably when controlling a simulated evacuee, and making scenario changes that address real concerns and scenarios of interest when acting as an overseer. This mode will require a high-degree of interpretation of the results produced and, by definition, of user interaction.

The data requirements fall somewhere between the Predictive and Engineered modes, depending on the exact nature of the simulation and the scope of the engineer interaction. The model will have to respond to the changes in the scenario conditions and have sufficient data to do this. The current scarcity of data may limit the scope of the interaction between the model and the user. A model will need to simulate the evacuees as individuals, accept user input during the simulation and allow evacuees to respond accordingly; i.e. have a sufficiently developed behavioral model to allow the simulated evacuees to do this. Models currently exist that are able to do this (e.g. EVI [10]). EVI is often used as a training tool, enabling trainees to directly interact with the evacuation scenario and more clearly see the effect of their actions.

\subsection{Real-Time Mode}

Real-Time mode allows the user to gain feedback from a model during an actual situation, be it emergency or non-emergency; i.e. to direct the real-life procedure employed as the situation evolves. This requires a direct feed from the observed real-life conditions to the conditions simulated within the egress model. This might be through a CCTV (closed-circuit television) system/sensor system/manual observation that monitor real-life events and 
then provide this information directly to the egress model. The model then simulates how the situation evolves. The operator would then be able to play the current situation forward allowing them to estimate how the current situation may develop given different procedural responses. This would need a link between the observed and simulated conditions and the model run-time to be significantly faster than real-time in order to allow the results to be produced and processed in time.

In some occupancies, Real-Time mode would be used in the normal operation of the building to assess current behavior and performance. Feedback could then be provided on the movement of people around the space and procedural modifications made if required. For instance, in high occupancy spaces, such as stadia, the comfort levels of people can be managed using Real-Time analysis. Procedures can be employed to maintain or improve comfort levels. These procedures would be better informed and tested through the use of Real-Time mode. In addition, Real-Time mode can be used to prevent the normal operations within stadia developing into emergency situations. In other occupancies, this type of use may not be warranted; e.g. in an office building. However, there may be value in assessing the response of the evacuating population and determining whether this can be improved in response to the evolving scenario. This could be of value to the evacuees, the building managers, and also to the first responders.

In Real-Time mode, the initial scenario conditions are determined by external sources. Where there are omissions, data can be provided from empirical data, from the use of Operational mode, or from the engineer. Otherwise, the configuration of the mode would be similar to those of Engineered mode: allowing key procedural changes to be assessed in faster than real-time. Currently, it is not feasible for the intensive analysis required by Predictive mode to be conducted under such time constraints.

In Real-Time mode, the engineer will be responsible for setting up the initial conditions and interpreting the results produced. It is critical that the engineer understands the model accuracy sacrificed to cater for real-time application: the results are generally indicative and may be further compromised due to time constraints. The engineer may also be under the same time constraints. The results provided may need to be analyzed quickly in order to provide feedback to the procedures implemented. This will require an intuitive understanding of the results and of the procedures available. Real-Time mode may be accompanied by analytical tools that assist the engineer in this process.

Several models are currently available that can run faster than real-time (one example is EVACNET [11]), although many of these do not have the sophistication to provide the necessary detailed feedback to the engineer. In addition, models exist that are designed specifically to operate in the manner described above (e.g. the model being produced by UTRC [12]), although there is currently little information on their capabilities. 


\section{Discussion}

Each of these modes provides an opportunity to examine different phases of people movement; i.e. one of the six combinations identified in Table 1. Currently, an engineer would require several simulation models in order employ all of these modes. However, in reality, these modes are highly coupled; i.e. the results from one will affect the conditions in another. Therefore, it would be highly beneficial if the modes were included in a single model that allowed information to migrate between the modes directly. Although it would be possible to port information between models, it is more prone to error; it is more likely that data will be lost in the translation; and it will invoke the cost of purchasing/training for several models.

An integrated model (i.e. one that includes all six modes and allows information to propagate between them) may be used in the following manner. An airport design is presented to an engineer prior to construction along with information on the use of the space; e.g. expected movement around an airport. The engineer employs Nä̈ve mode in order to investigate how the airport configuration influences people movement. Issues and recommendations are passed on to the architects. Once the design is confirmed the engineer is asked to assess egress performance as part of a performance-based design (PBD). The engineer first employs Predictive mode to identify key scenario conditions. This is based on the calculations in Nä̈ve mode and on the limited empirical data available. Engineered mode is then employed to conduct the standard PBD assessment, where specific scenario questions are answered by the simulation model. Guidance may also be provided on the procedural design. Once the design has been accepted then the model can be used in Operational mode to optimize the nonemergency procedures employed; e.g. checking in, security issues, etc. This will be influenced by the results produced by Naïve mode. This will identify critical egress components, exit familiarity and also how the population is distributed. In order to improve (non-)emergency preparedness, the model can be employed in Interactive mode. As part of their training staff may engage with the model on an individual or global level giving them first-hand insight into the scenario and the procedural response. Once the building is operational, the model could be running in Real-Time mode, with information from CCTV cameras, providing safety managers with some indication as the effectiveness of different procedures. If an incident does occur, the model can be employed in Engineered and Predictive modes to investigate the behavioral/procedural response and recommend improvements to it.

Although it is improving, the application of computational egress models is still relatively immature, especially in comparison with comparable approaches such as the use of computational fluid dynamics (CFD) models. This may produce problems, especially given the broader range of application types and the increased level of expertise required. It is often assumed that expertise from the traditional hydraulic calculations can be translated directly into the computational arena. Although experience in the application of hydraulic models may be necessary for applying egress models, it is certainly not sufficient. This becomes all the more evident as the 
project applications become more complex and require a greater understanding of human behavior in fire.

\section{Conclusion}

Six modes of application have been presented. These broadly reflect the different approaches that are available to simulation models when examining people movement. These require different levels of model development, data, and user expertise. Although this is not an exhaustive list of modes, it does demonstrate the variability in the use of the simulation models and the impact on the user and on the data required. It also demonstrates the interdependence of these modes, given their highly coupled nature.

All of these modes have great value; however, currently no model can address all of them. This will inevitably require the engineer to make a greater number of assumptions. Incorporating all of these modes into a single model would produce a number of benefits. The engineer would be able to represent the impact of occupant experience, the different phases of people movement and the interaction between the procedures given a situation. This should be a future goal, given the need for this integrated approach and the increasing interrelatedness of incident scenarios. It would also implicitly encourage the view of the building as a highly coupled people movement system rather than as a set of distinct situations that can be examined in isolation.

\section{References}

[1] Boswell, D., and Gwynne, S.M.V., Air, Fire and ICE: Fire \& Security Challenges Unique to Airports, to appear Fire and Security Today, August, 2007.

[2] Sime, J, Escape Behaviour In Fire: 'Panic' Or Affiliation?, PhD Thesis, Department Of Psychology, University Of Surrey, 1984.

[3] Turner, A. and Penn, A., Encoding natural movement as an agent-based system: an investigation into human pedestrian behaviour in the built environment. Environment and Planning B: Planning and Design 29(4):473490, 2002.

[4] Xie, H., Filippidis, L., Galea E.R., Gwynne S., Blackshields, D., Experimental Study and theoretical analysis of signage legibility distances as a function of observation angle, Proc Pedestrian and Evacuation Dynamics 2005, Ed: Waldau, N., Gattermann, P., Knoflacher, H., Schreckenberg, M., Springer, Germany, ISBN 878-3-540-47062-5, pp131-143, 2007.

[5] Pan, X., Computational Modeling of Human and Social Behaviors For Emergency Egress Analysis, PhD Dissertation, Dept. Civil and Environmental Engineering, Stanford University, 2006.

[6] Paulsen, T., Soma, H., Schneider, V., Wiklund, J., Lovas, G., Evaluation Of Simulation Models Of Evacuation From Complex Spaces, SINTEF Report, STF75 A95020, ISBN 82-595-8583-9, June 1995.

[7] Meacham, B.J. and Custer, R.L.P., Performance-Based Fire Safety Engineering: an Introduction of Basic Concepts, Journal of Fire Protection Engineering, Vol. 7, No. 2, 35-53, 1995. 
[8] Kuligowski, E.D., and Gwynne S.M.V., What a user should know about selecting an evacuation model, Fire Protection Engineering Magazine, Human Behaviour in Fire Issue, Fall, 2005.

[9] Thompson, P. And Marchant, E., A Computer Model For The Evacuation Of Large Building Populations, Fire Safety Journal, 24, pp131-148,1995.

[10] Vassalos, D., Kim, H., Christiansen, G., Majumder, J., A Mesoscopic Model for Passenger Evacuation in a Virtual Ship-Sea Environment and Performance-Based Evaluation Pedestrian and Evacuation Dynamics, ed: Schreckenberg, M. and Sharma, S.D., ISBN 3540426906, 2001, pp369-391. [11] Kisko, T.M. And Francis, R.L., Evacnet+: A Computer Program To Determine Optimal Evacuation Plans, Fire Safety Journal,9,pp211-220,1985. [12] Presented by UTRC at Pedestrian Evacuation Dynamics 2008. 